\title{
Supramolecular reorientation during deposition onto metal surfaces of quasi-two-dimensional Langmuir monolayers composed from bifunctional amphiphilic, twisted perylenes
}

\author{
Maximilian L. Hupfer ${ }^{1,2}$, Robert Meyer ${ }^{1,2}$, Tanja Deckert-Gaudig ${ }^{1,2}$, Soumik Ghosh ${ }^{1,2,5}$, Artem Skabeev ${ }^{3}$, \\ Kalina Peneva ${ }^{3}$, Volker Deckert ${ }^{1,2,6}$, Benjamin Dietzek ${ }^{1,2}$, Martin Presselt ${ }^{1,4,5, *}$ \\ ${ }^{1}$ Leibniz Institute of Photonic Technology (IPHT), Albert-Einstein-Str. 9, 07745 Jena, Germany \\ ${ }^{2}$ Institute of Physical Chemistry and Abbe Center of Photonics, Friedrich Schiller University Jena, \\ Helmholtzweg 4, 07743 Jena, Germany. \\ ${ }^{3}$ Institute of Organic and Macromolecular Chemistry, Friedrich Schiller University Jena, \\ Humboldtstraße 10, 07743 Jena, Germany. \\ ${ }^{4}$ Center for Energy and Environmental Chemistry Jena (CEEC Jena), Friedrich Schiller University Jena, \\ Philosophenweg 7a, 07743 Jena, Germany. \\ ${ }^{5}$ sciclus GmbH \& Co. KG, Moritz-von-Rohr-Str. 1a, 07745 Jena, Germany. \\ ${ }^{6}$ Institute of Quantum Science and Engineering, Texas A\&M University, College Station, TX 77843- \\ 4242, USA \\ *Corresponding author: martin.presselt@leibniz-ipht.de
}




\section{SYNTHESIS}

General methods: NMR spectra were recorded on a Bruker Avance 300. ${ }^{1} \mathrm{H}$ spectra were referenced to residual solvent signals $\left(\mathrm{C}_{2} \mathrm{D}_{2} \mathrm{Cl}_{4}\right)$, chemical shifts are reported in parts per million (ppm). Mass spectrometry was carried out with a Thermo Fisher UHPLC-Orbitrap Q-Extractive plus (APCI). Elemental analysis was performed with a VARIO EL III CHNS from Elementaranalysensysteme GmbH Hanau (Germany).

Materials: All solvents and chemicals were purchased in commercial grade (Acros, Alfa Aeser, Deutero, Eurisotop, Fisher, Grüssing, Merck, Roth, Sigma Aldrich, TCl and VWR) and unless otherwise stated, used as obtained. The progress of all reactions was monitored by thin-layer chromatography (Merck 60 F254). Column chromatography was performed with MachereyNagel silica gel 60 (grain size 0.04-0.063 nm).

\section{9,10-bis([1,2]dithiole)-1,6,7,12-tetrachloroperylene monoanhydride \%compoundnumber\% ${ }^{1}$}<smiles>O=c1oc(=O)c2c3c(Br)cc(Cl)c2c2c1cc(Cl)c1c(c(Cl)cc3Br)c12</smiles>

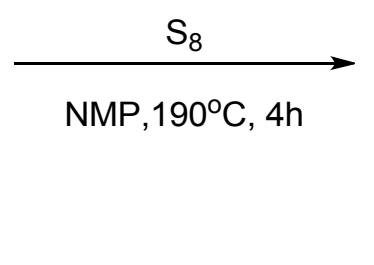<smiles></smiles>

A suspension of 9,10-tetrabromo-1,6,7,12-tetrachloroperylene monoanhydride $(0.617 \mathrm{~g}$, $1.00 \mathrm{mmol})$ and sulfur $(0.13 \mathrm{~g}, 4.0 \mathrm{mmol})$ in $40 \mathrm{ml} \mathrm{NMP}$ was stirred at $190^{\circ} \mathrm{C}$ for $4 \mathrm{~h}$. After cooling down to room temperature the reaction mixture was poured $200 \mathrm{ml} 0.1 \mathrm{M} \mathrm{HCl}$ solution. Formed precipitate was filtered, washed with water and dried. The crude product

${ }^{1}$ According literature procedure: Org. Lett. 2014, 16, 11, 2814-2817 (DOI: 10.1021/ol5008586) 
was purified by column chromatography using dichloromethane as eluent on silica. Yield 500 $\mathrm{mg}(96 \%)$.

${ }^{1} \mathrm{H}-\mathrm{NMR}\left(300 \mathrm{Mhz}, \mathrm{C}_{2} \mathrm{D}_{2} \mathrm{Cl}_{4}, 300 \mathrm{~K}\right): 8.60(\mathrm{~s}, 2 \mathrm{H}) ; 7,64(\mathrm{~s}, 2 \mathrm{H})$.

APCI-MS (pos.) m/z (\%) [M+H]: calc. 522.840; found 522.840 (100)

Elemental analysis: calc.: \% C 50.60; \% H 0.77; \% Cl 27.15; \% S 12.28. found: \% C 50.03; \% H $1.01 ; \% \mathrm{Cl} 27.35 ; \mathrm{S} 12.05$.

\section{$\Pi(A)$ ISOTHERMS}

In the following figures, the extrapolation procedure to determine $A_{0}$ areas are graphically shown. Therefore, averaged isotherms have been used. Conditions at spreading the perylene solutions: $\mathrm{c}_{\text {spread }}=0.02 \mu \mathrm{mol} / \mathrm{ml}, 20^{\circ} \mathrm{C}$ subphase temperature, and $5 \mathrm{ml}$ spreading volume.

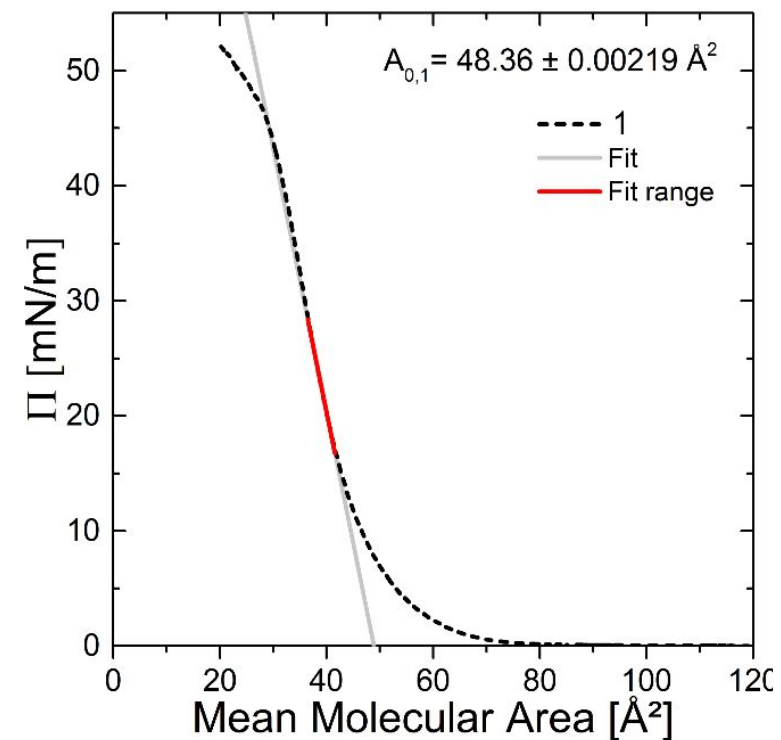

Figure S 1: Mean $\Pi(A)$ isotherm (black, dotted) and extrapolation to yield the $A_{0}$-area (grey) of compound 1

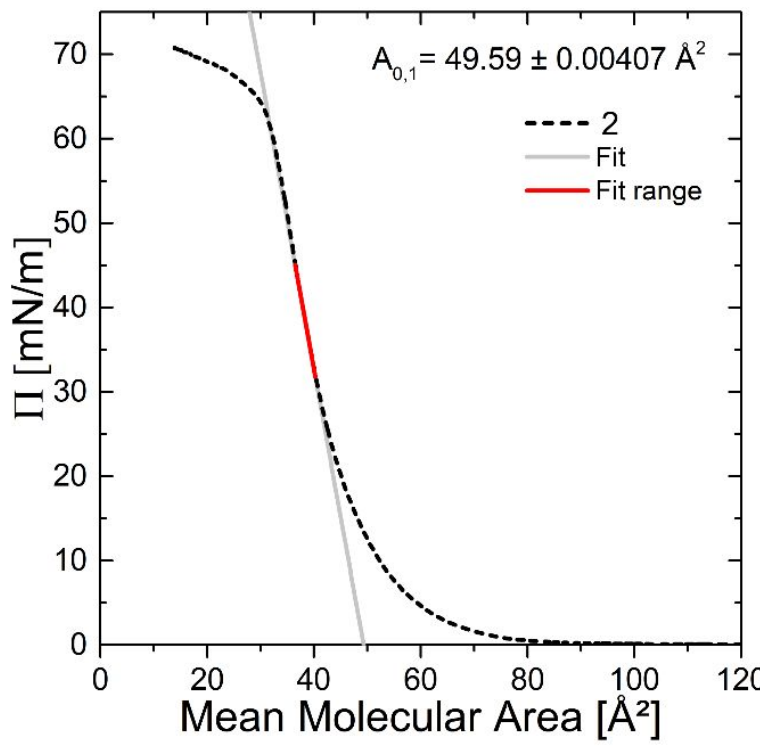

Figure S 2: Mean $\Pi(A)$ isotherm (black, dotted) and extrapolation to yield the $A_{0}$-area (grey) of compound 2 


\section{MODELLING MONOMER AND DIMER PROPERTIES}

We used the program Energyscan ${ }^{1}$ to identify energetically favorable and geometrically diverse dimer structures. These calculations were carried out for a square grid of side $25 \AA$ for compound $\mathbf{1}$ and of side $50 \AA$ for compound $\mathbf{2}$ with grid points every $0.5 \AA$. At each grid point, molecules were rotated in all three axes (Figure SI 3), at 12-degree intervals. Then we isolated geometrically diverse structures with RMSD of 0.1 from each other and within an energy range of $250 \mathrm{meV}$ of the global minima. If sufficient structures were not found, we reduced the RMSD threshold by 0.05 until enough structures were found. 


\section{Translational Grid}
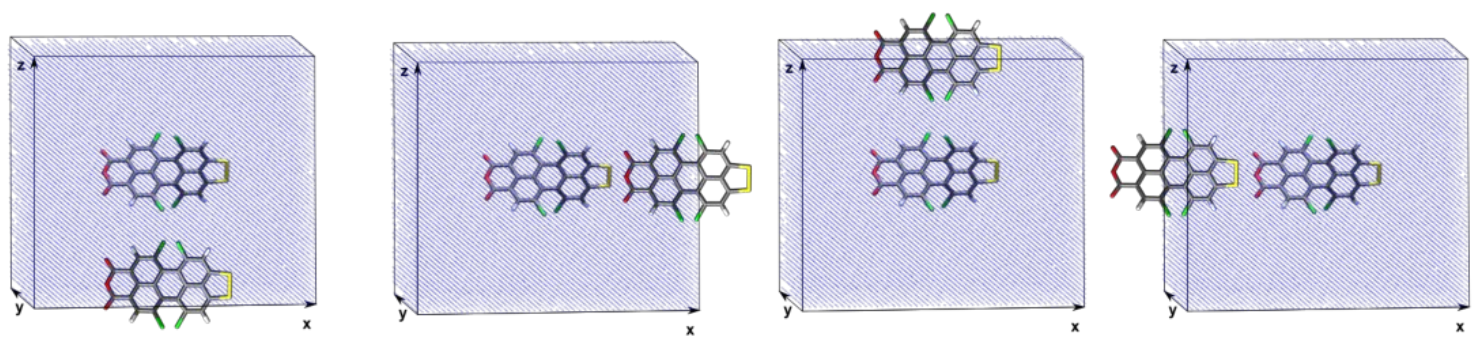

\section{Rotational Grid}
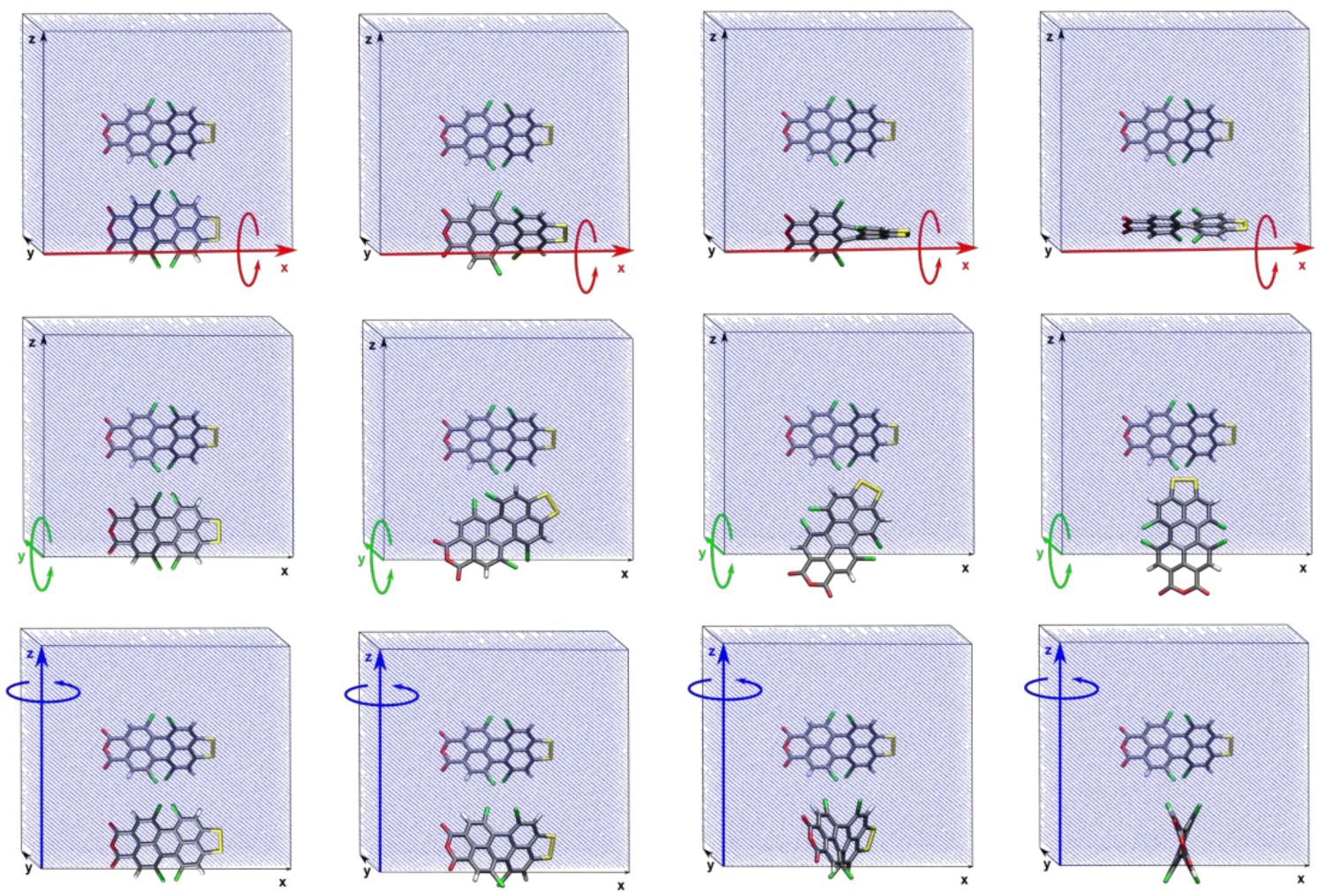

Figure S 3: Model dimer search for compound 1. It includes dimers creatable by just translation at each grid point (Row 1) and rotations of $0,30,60,90$ degrees each along $X$ (row 2), $Y$ (row 3 ) and $Z$ (row 4 ) axes at each grid point. In our calculations we extended this search for the entire grid and for a full 360 degrees rotation

\section{DENSITY FUNCTIONAL THEORY CALCULATIONS}

Once the dimers were selected on the basis of their uff forcefield energy, all selected dimers underwent quantum chemical structure optimizations and calculations of electrostatic potential using 
density functional theory (DFT) as implemented in the GPU-accelerated program TeraChem ${ }^{2-5}$ with the LANL2DZ ${ }^{6}$ basis set and hybrid functional B3LYP7. Thus, binding energies with respect to the monomer were calculated. We also calculated the degree of change caused due to the DFToptimization. These are represented by the axes Binding Energy (eV) and RMSD ( $\AA$ ) in Figure SI 4-5. Then the most stable dimers were taken for further calculations at the COSMO conductor limit. Grimme's D3 dispersion correction ${ }^{8,9}$ has been used for all calculations. Electrostatic potentials were plotted on the electron density isosurface of 0.0004 a.u. 


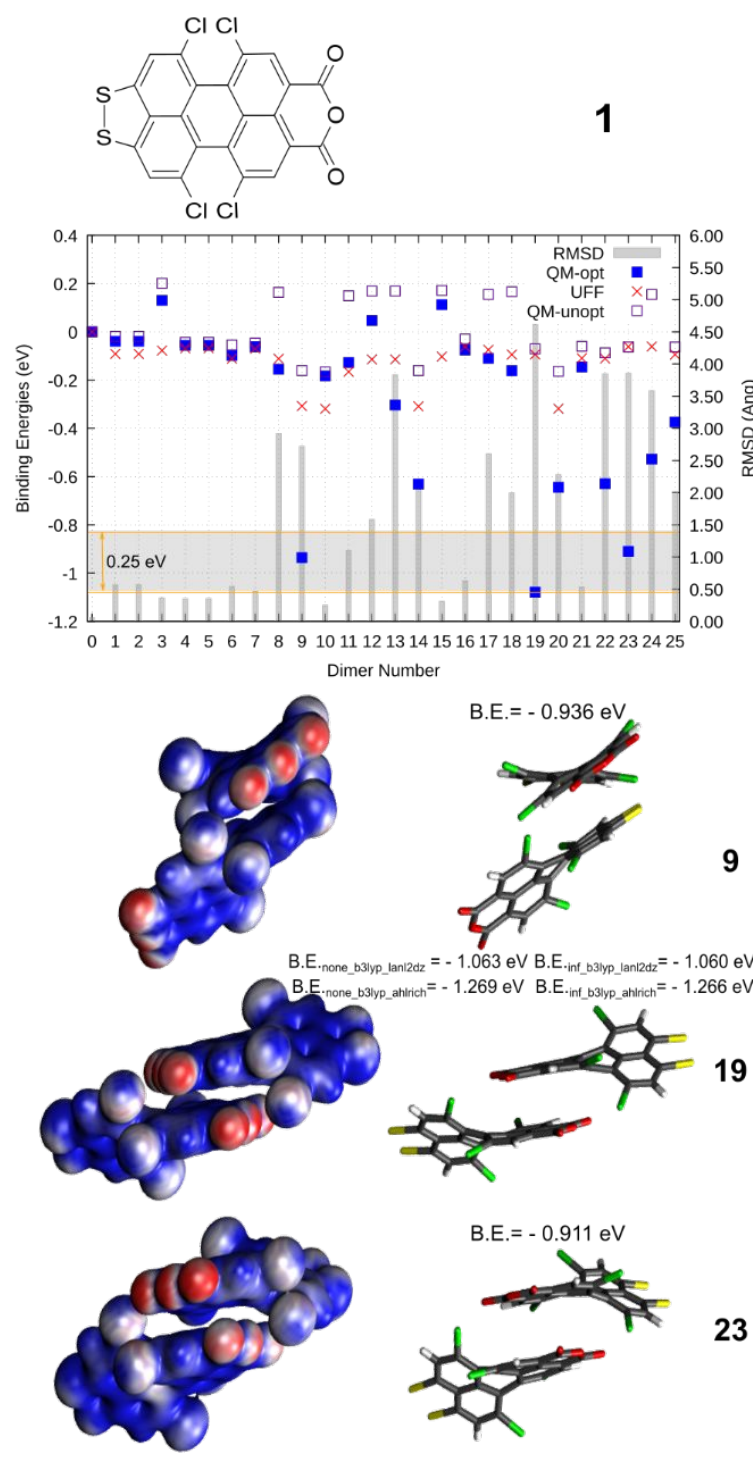

Figure S 4: Binding energy comparisons between force field (uff single point calculation) and QM (b3lyp, lanl2dz_ecp) calculations for perylene 1 for different dimers ( 0 denotes the monomer). Dimers with lowest QM energy (dimers 9, 19 and 23) were taken for further theory calculations. "none" and "inf" denote vacuum and conductor environment accounted for by setting the permittivity constant to Zero and to Infinity as parameters in the COSMO model.

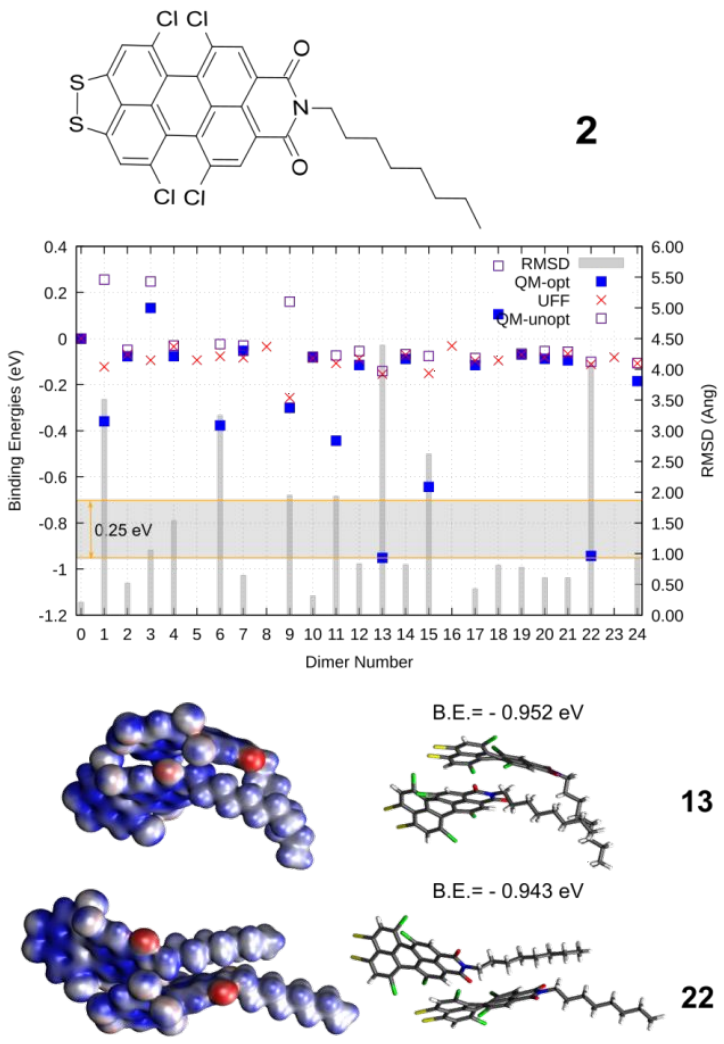

Figure S 5: Binding energy comparisons between force field (uff single point calculation) and QM (b3lyp, lanl2dz_ecp) calculations for perylene 2 for different dimers ( 0 denotes the monomer). Dimers with lowest QM energy (dimers 13 and 22) were taken for further theory calculations. "none" and "inf" denote vacuum and conductor environment accounted for by setting the permittivity constant to Zero and to Infinity as parameters in the COSMO model. 


\section{AFM DATA}

Table S 1: Root mean square (RMS) roughness derived from scanning force microscopy measurements of LB films from perylene 1 (left) and 2 (right) at LB and LS deposition pressures of $25 \mathrm{mN} / \mathrm{m}$ on glass and gold with different deposition directions (up $\uparrow$, down $\downarrow$, down-up $\downarrow \uparrow$ )

\begin{tabular}{|c|c|c|c|c|c|}
\hline \multicolumn{3}{|c|}{ Perylene 1} & \multicolumn{3}{|c|}{ Perylene $\mathbf{2}$} \\
\hline \multicolumn{2}{|c|}{ Deposition } & $\begin{array}{l}\text { RMS roughness } \\
{[\AA ̊]}\end{array}$ & \multicolumn{2}{|c|}{ Deposition } & $\begin{array}{l}\text { RMS roughness } \\
{[\AA ̊]}\end{array}$ \\
\hline glass & $\uparrow$ & 19.62 & glass & $\uparrow$ & 7.18 \\
\hline \multirow[t]{4}{*}{ gold } & $\uparrow$ & 6.32 & gold & $\uparrow$ & 6.88 \\
\hline & $\downarrow$ & 7.13 & & $\downarrow$ & 6.60 \\
\hline & $\downarrow \uparrow$ & 6.46 & & $\downarrow \uparrow$ & 7.38 \\
\hline & LS & 6.66 & & LS & 8.93 \\
\hline
\end{tabular}

Table S 2: Root mean square (RMS) roughness derived from scanning force microscopy measurements of the blank substrates

\begin{tabular}{|l|l|}
\hline Blank & RMS roughness $[\AA]]$ \\
\hline Quartz glass & 5.60 \\
\hline Gold & 7.58 \\
\hline
\end{tabular}



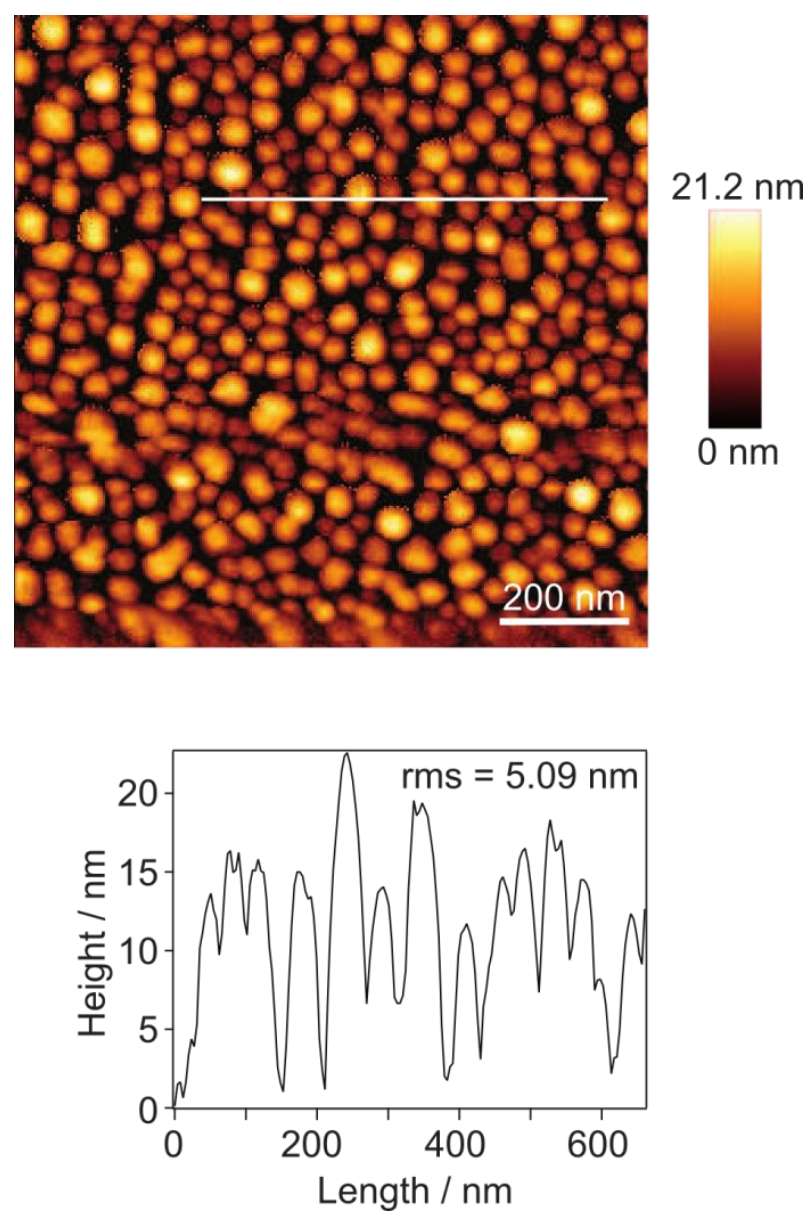

Figure S 6: AFM topography (top) of a silver island film used for the present SERRS experiments. The white line indicates where the profile line (bottom) and the rms of the height distribution as measure of the roughness across the substrate were detected. 


\section{SERRS}

Table S 3: Band assignment of SERRS spectra of 1 and $\mathbf{2}$
Perylene
Assignment
Perylene
Assignment

anhydride (1)

monoimide (2)

$1775 \quad \mathrm{C}=\mathrm{O} \operatorname{str}^{10-12}$

1592

$\mathrm{C}=\mathrm{C} \operatorname{str}^{11,12}$,

1706

$\mathrm{C}=\mathrm{O}^{13-17}$

1593

$\mathrm{C}=\mathrm{C} \operatorname{str}^{13,19}$,

perylene ring $\operatorname{str}^{18}$

perylene ring $\operatorname{str}^{14,16,17,20,21}$

1564

$\mathrm{C}=\mathrm{C} \operatorname{str}^{10,11}$

1540

$\mathrm{C}=\mathrm{C}-\mathrm{Cl} \operatorname{str}^{15}$

1536

$\mathrm{C}=\mathrm{C}-\mathrm{Cl} \operatorname{str}^{15}$

1470

perylene ring $\operatorname{str}^{16,17}$

1433

$C=C^{14,15}$, perylene ring $s^{22}$

1417,1426

C-H def, C-C str ${ }^{10}$

1388

1343

$\mathrm{C}-\mathrm{H} \operatorname{def}^{10}, \mathrm{C}-\mathrm{C} \operatorname{str}^{10}$

1333

1317

1091

perylene ring $\operatorname{str}^{18}, \quad 1055$

1062

\author{
C-O-C, C-C, C-H def ${ }^{10,12}$
}

perylene ring $\operatorname{str}^{13,16,17,20,21}$

C-N str" ${ }^{13,19}$, C-H (chain) ${ }^{15,16}$

$\mathrm{C}-\mathrm{H}$ def, perylene ring $\operatorname{str}^{13}$, $16,17,19-21$

C-H def ${ }^{13}, 14,16,17,19,20$

C-H def ${ }^{13,22}$ 
910, 923

$$
\text { C-C-Cl str'23, 24? }
$$

898

$\mathrm{C}-\mathrm{C}-\mathrm{H}$ def ${ }^{10}, \mathrm{C}-\mathrm{C}-\mathrm{Cl}{ }^{23}$ ?

758

C-H def ${ }^{14,19}$

706

$$
\text { C-S } 25
$$

712

C-S 25

620

$\mathrm{C}-\mathrm{C}-\mathrm{C}, \mathrm{C}-\mathrm{C}=\mathrm{O}^{10}$

568

$\mathrm{C}-\mathrm{Cl}^{23}$ ?

569

$\mathrm{C}-\mathrm{H} \operatorname{def}^{13}, \mathrm{C}-\mathrm{Cl}^{23}$ ?

544

perylene ring def ${ }^{18}$,

546

perylene ring def ${ }^{14,16,19-22,}$

$\mathrm{C}-\mathrm{C} \operatorname{str}^{10}, \mathrm{C}-\mathrm{Cl}{ }^{23}$ ?

$\mathrm{C}-\mathrm{Cl}^{23} ?$

489

C-C-C, C-C-H def ${ }^{10}$

494

ring def ${ }^{13}$

422,446

C-O-C, C-C $=0$ def ${ }^{10}$

426

?

382

C-O-C, C $=0$ def ${ }^{10}$

252

$$
\mathrm{Ag}-\mathrm{S}^{25}
$$

248

Ag-S 25

\section{TRANSFER RATIO}

Table S 4: Transfer ratio (TR) of LB films from perylene 1 (left) and 2 (right) at LB deposition pressures $\left(\Pi_{\mathrm{LB}}\right)$ of $25 \mathrm{mN} / \mathrm{m}$ on glass, gold and silver with different deposition directions (up $\uparrow$, down $\downarrow$, down-up $\downarrow \uparrow$ )

\begin{tabular}{|l|l|l|l|l|l|}
\hline \multicolumn{2}{|l|}{ Perylene 1 } & \multicolumn{2}{l|}{ Perylene 2 } \\
\hline \multicolumn{2}{|l|}{ Deposition } & Transfer ratio & \multicolumn{2}{l|}{ Deposition } & Transfer ratio \\
\hline glass & $\uparrow$ & 1.23 & glass & $\uparrow$ & 0.78 \\
\hline gold & $\uparrow$ & 0.4 & gold & $\uparrow$ & 0.98 \\
\hline
\end{tabular}




\begin{tabular}{|l|l|l|l|l|l|}
\hline & $\downarrow$ & 0.53 & & $\downarrow$ & 0.66 \\
\hline & $\downarrow \uparrow$ & 0.53 and 0.4 & & $\downarrow \uparrow$ & 0.66 and 0.03 \\
\hline silver & $\uparrow$ & $/$ & silver & $\uparrow$ & 0.54 \\
\hline
\end{tabular}




\section{REFERENCES}

1. Sachse, T.; Martinez, T. J.; Dietzek, B.; Presselt, M., A program for automatically predicting supramolecular aggregates and its application to urea and porphin. Journal of computational chemistry 2018, 39 (13), $763-772$.

2. Isborn, C. M.; Luehr, N.; Ufimtsev, I. S.; Martínez, T. J., Excited-State Electronic Structure with Configuration Interaction Singles and Tamm-Dancoff Time-Dependent Density Functional Theory on Graphical Processing Units. Journal of Chemical Theory and Computation 2011, 7 (6), 1814-1823.

3. Song, C.; Wang, L.-P.; Martínez, T. J., Automated Code Engine for Graphical Processing Units: Application to the Effective Core Potential Integrals and Gradients. Journal of Chemical Theory and Computation 2016, 12 (1), $92-106$.

4. Titov, A. V.; Ufimtsev, I. S.; Luehr, N.; Martinez, T. J., Generating Efficient Quantum Chemistry Codes for Novel Architectures. Journal of Chemical Theory and Computation 2013, 9 (1), 213-221.

5. Ufimtsev, I. S.; Martinez, T. J., Quantum Chemistry on Graphical Processing Units. 3. Analytical Energy Gradients, Geometry Optimization, and First Principles Molecular Dynamics. Journal of Chemical Theory and Computation 2009, 5 (10), 2619-2628.

6. Wadt, W. R.; Hay, P. J., Ab initio effective core potentials for molecular calculations. Potentials for main group elements Na to Bi. The Journal of Chemical Physics 1985, 82 (1), 284-298.

7. Stephens, P. J.; Devlin, F. J.; Chabalowski, C. F.; Frisch, M. J., Ab-Initio Calculation of Vibrational Absorption and Circular-Dichroism Spectra Using Density-Functional Force-Fields. The Journal of Physical Chemistry 1994, 98 (45), 1162311627.

8. Grimme, S.; Antony, J.; Ehrlich, S.; Krieg, H., A consistent and accurate ab initio parametrization of density functional dispersion correction (DFT-D) for the 94 elements H-Pu. The Journal of Chemical Physics 2010, 132 (15), 154104.

9. Das, S.; Fiedler, J.; Stauffert, O.; Walter, M.; Buhmann, S. Y.; Presselt, M., Macroscopic quantum electrodynamics and density functional theory approaches to dispersion interactions between fullerenes. Phys Chem Chem Phys 2020, 22 (40), 23295-23306.

10. Kobitski, A. Y.; Scholz, R.; Zahn, D. R. T., Theoretical studies of the vibrational properties of the 3,4,9,10-perylene tetracarboxylic dianhydride (PTCDA) molecule. Theo. Chem. 2003, 625, 39-46.

11. Zhanpeisov, N. U.; Nishio, S.; Fukumura, H., Density functional theory study of vibrational properties of the 3,4,9,10-perylene tetracarboxylic dianydride (PTCDA) molecule: IR, Raman, and UV-vis spectra. Int. J. Quant. Chem. 2005, $105,368-375$.

12. Saeed, A.; G., S., Synthesis, characterization and fluorescence studies of novel tetrachloropherylene-azo hybid dyes. J. Fluoresc. 2014, 24, 1337-1345.

13. Rodriguez-Llorente, S.; Aroca, R.; Duff, J., Vibrational spectra and thin solid films of a bi(propylperylenediimide). J. Mater. Chem. 1998, 8 (10).

14. Rodriguez-Llorente, S.; Aroca, R.; Duff, J.; de Saja, J. A., Spectroscopic characerization of thin solid films of 1,3 bis(3-chlorobenzylimido perylene) propane. Thin Solid Films 1998, 317, 129-132.

15. Tenori, E.; Colusso, A.; Syrgiannis, Z.; Bonasera, A.; Osella, S.; Ostric, A.; Lazzaroni, R.; Megeghetti, M.; Prato, M., Perylene derivatives as usefuls SERRS reporters, including multiplexing analysis. ACS Appl. Mat. Inter. 2015, 7, 2804328048.

16. Rodriguez-Llorente, S.; Aroca, R.; Duff, J., Spectroscopic characterization of thin solid films of a bis(chlorobenzylimidoperyleneimido)octane derivative. J. Mater. Chem. 19978, 8 (3), 629-632.

17. Kam, A. P.; Aroca, R., Perylene tetracarbocylic-phthalocyanine mixed thin solid films. Surface-enhanced resoncance Raman scattering imaging studies. Chem. Mater. 2001, 13, 4463-4468.

18. Jennings, C. A.; Kovacs, G., . J.; Aroca, R., Fourier transform surface-enhanced Raman scattering of LangmuirBlodgett monolayers on copper and gold island substrates. Langmuir 1993, 9, 2151-2155.

19. Del Cano, T.; Duff, J.; Aroca, R., Molecular spectra and molecular organization in thin solid films of bis(neopentylimido) perylen. Appl. Spectrosc. 2002, 56 (6), 744-750.

20. Aroca, R.; Constantino, C. J. L., Surface-enhanced Raman scattering: imaging and mapping of Langmuir-Blodgett monolayers physically adsorbed onto silver island films. Langmuir 2000, 16, 5425-5429.

21. Johnson, E.; Aroca, R.; Nagao, Y., Electronic and vibrational spectra of films and Langmuir-Blodgett monolayers of N-octyl-3,4-perylenedicarboxidimide. J. Phys. Chem. 1991, 95, 8840-8843.

22. Constantino, C. J. L.; Aroca, R., Surface-enhanced resonance Raman scattering imaging of Langmuir-Blodgett monolayers of bis(benzimidazo)perylene on silver island films. J. Raman Spectrosc. 2000, 31, 887-890.

23. Neugebauer, J.; Baerends, E. J., Combined theoretical and experimental deep-UV resonance Raman studies of substituted pyrenes. J. Phys. Chem. A 2005, 109, 2100-2106.

24. Arivazhagan, M.; Gayathri, R., NBO, NMR, UV, FT-IR, FT-Raman specra and molecular structure (monomeric and dimeric structures) investigation of 4-Chloro-3,5-Xylenol: a combined expoerimental and theoretical study. Spectrochim. Acta A 2013, 116, 170-182. 
25. Nyamekye, C. K. A.; Weibel, S. C.; Smith, E. A., Directional Raman scattering spectra of metal-sulfur bonds at smooth gold and silver substrates. J. Raman Spectrosc. 2020, 52, 1246-1255. 